\title{
The b quark mass from lattice nonrelativistic QCD
}

\author{
Alistair Hart ${ }^{a}$, Georg M. von Hippel ${ }^{b}$, R. R. Horgan ${ }^{c}$, Andrew Lee ${ }^{c}$, Christopher J. \\ Monahan*c \\ ${ }^{a}$ SUPA, School of Physics and Astronomy, University of Edinburgh, Edinburgh EH9 3JZ, U.K. ${ }^{\dagger}$ \\ ${ }^{b}$ Institut für Kernphysik, Universität Mainz, Becherweg 45, 55099 Mainz, Germany \\ ${ }^{c}$ DAMTP, University of Cambridge, Wilberforce Road, Cambridge CB3 OWA, U.K. \\ Email: C.Monahan@damtp.cam.ac.uk, a.hart@ed.ac.uk, \\ hippel@kph.uni-mainz.de, R.R.Horgan@damtp.cam.ac.uk, \\ A. Lee@damtp.cam.ac.uk
}

We present the first two-loop calculation of the heavy quark energy shift in lattice nonrelativistic QCD (NRQCD). This calculation allow us to extract a preliminary prediction of $m_{b}\left(m_{b}, n_{f}=\right.$ $5)=4.25(12) \mathrm{GeV}$ for the mass of the $\mathrm{b}$ quark from lattice NRQCD simulations performed with a lattice of spacing $a=0.12 \mathrm{fm}$. Our result is an improvement on a previous determination of the $b$ quark mass from unquenched lattice NRQCD simulations, which was limited by the use of one-loop expressions for the energy shift. Our value is in good agreement with recent results of $m_{b}\left(m_{b}\right)=4.163(16) \mathrm{GeV}$ from QCD sum rules and $m_{b}\left(m_{b}, n_{f}=5\right)=4.170(25) \mathrm{GeV}$ from realistic lattice simulations using highly-improved staggered quarks. We employ a mixed strategy to simplify our calculation. Ghost, gluon and counterterm contributions to the energy shift and mass renormalisation are extracted from quenched high-beta simulations whilst fermionic contributions are calculated using automated lattice perturbation theory. Our results demonstrate the effectiveness of such a strategy.

The XXVIII International Symposium on Lattice Field Theory, Lattice2010

June 14-19, 2010

Villasimius, Italy

\footnotetext{
${ }^{*}$ Speaker.

${ }^{\dagger}$ Current address: Cray Exascale Research Initiative, JCMB, King’s Buildings, Edinburgh EH9 3JZ, U.K.
} 


\section{Introduction}

The precise theoretical and experimental determination of quark masses is an important component of high-precision tests of the Standard Model of particle physics. One current focus for tests of the Standard Model is the unitarity of the Cabibbo-Kobayashi-Maskawa (CKM) matrix, which describes flavour-changing quark transitions. Quark masses serve as an input into the tests of CKM matrix unitarity; the mass of the b quark is used in the extraction of the CKM matrix element $\left|V_{u b}\right|$ from inclusive semileptonic decays of B mesons [1].

Recent high-precision calculations of the b quark mass using realistic lattice QCD simulations [2] and perturbative QCD combined with experimental results [3] are in good agreement, obtaining values of $m_{b}\left(m_{b}, n_{f}=5\right)=4.170(25) \mathrm{GeV}^{1}$ and $m_{b}\left(m_{b}\right)=4.163(16) \mathrm{GeV}$ respectively. For the first time, the lattice result was obtained using the same action, the highly improved staggered quark (HISQ) action, for both valence and sea quarks. HISQ is a highly corrected version of the standard staggered quark action that retains a chiral symmetry on the lattice [4]. Most current lattice studies of b quarks use an effective field theory, such as nonrelativistic QCD (NRQCD), for the valence heavy quark. Simulating both valence and sea quarks with the same action allows a much greater precision, but is only now becoming possible with the advent of finer lattices and highly improved actions. However, even on the very finest lattices with HISQ heavy quarks an extrapolation to the heavy quark mass is still required [2].

Our calculation improves on a previous determination of $m_{b}\left(m_{b}\right)=4.4(3) \mathrm{GeV}$ from unquenched lattice QCD simulations using NRQCD valence $b$ quarks [5]. The dominant error in that calculation arose from the use of one-loop perturbation theory in the matching between lattice quantities and the continuum result. By introducing a mixed strategy incorporating high-beta quenched simulations and automated lattice perturbation theory, we perform the first ever such twoloop calculation in NRQCD. This serves a two-fold purpose. Firstly our calculation demonstrates the effectiveness of employing an efficient mixed perturbation theory/high-beta simulation method for higher order perturbative quantities. Secondly our result allows us to obtain a more precise prediction for the $b$ quark mass from lattice NRQCD simulations.

\subsection{Heavy quarks on the lattice}

Currently available lattices are too coarse to directly simulate b quarks, because the Compton wavelength of the $b$ quark is smaller than the lattice spacing. One common approach to solving this problem is to introduce a nonrelativistic effective action, NRQCD, for which the discretization errors are under control and which can be systematically improved by including extra operators.

NRQCD is constructed by integrating out dynamics at the scale of the heavy quark mass and then using the Foldy-Wouthuysen-Tani transformation to write the action as an expansion in the inverse heavy quark mass [6]. We use an NRQCD action correct to $\mathscr{O}\left(1 / m^{2}, v^{4}\right)$, where $v$ is the relative internal velocity of the bound-state heavy quarks. A detailed derivation of the action we use is given in [7]. The lattice NRQCD action can be written

$$
S_{\mathrm{nrqcd}}=\sum_{\mathbf{x}, \tau} \psi^{+}(\mathbf{x}, \tau)[\psi(\mathbf{x}, \tau)-K(\tau) \psi(\mathbf{x}, \tau-1)],
$$

\footnotetext{
${ }^{1}$ Note that Reference [2] quotes only the result at a scale equal to $10 \mathrm{GeV}$ and the value of $m_{b}\left(m_{b}, n_{f}=5\right)$ was obtained using continuum perturbation theory for the running $\overline{\mathrm{MS}}$ mass.
} 
with

$$
K(\tau)=\left(1-\frac{\delta H}{2}\right)\left(1-\frac{H_{0}}{2 n}\right)^{n} U_{4}^{\dagger}\left(1-\frac{H_{0}}{2 n}\right)^{n}\left(1-\frac{\delta H}{2}\right) .
$$

Here the leading nonrelativistic kinetic energy is $H_{0}=-\Delta^{(2)} / 2 M$. The correction term $\delta H$ contains higher order terms in the $1 / M$ expansion: the improved chromoelectric and chromomagnetic interactions, the leading relativistic kinetic energy correction and discretization error corrections. The integer $n$ is introduced as a stability parameter.

\section{Calculating the b quark mass}

Quark confinement ensures that quark masses are not physically measurable quantities, so the notion of quark mass is a theoretical construction. A wide range of quark mass definitions exist, often tailored to exploit the physics of each particular process. One common choice of quark mass is the pole mass, defined as the pole in the renormalized heavy quark propagator. However, the pole mass is a purely perturbative concept and suffers from infrared renormalon ambiguities [8-10]. To avoid these ambiguities, experimental results are usually quoted in the modified Minimal Subtraction $(\overline{\mathrm{MS}})$ scheme, which is renormalon ambiguity free. Lattice calculations use the renormalon-free bare lattice mass. These different quark mass definitions must be matched to enable meaningful comparison. We match bare lattice quantities to those in the $\overline{\mathrm{MS}}$ scheme using the pole mass as an intermediate step. Any renormalon ambiguities cancel in the full matching procedure between the lattice quantities and the $\overline{\mathrm{MS}}$ mass. We extract the $\overline{\mathrm{MS}}$ mass from lattice simulation data in a two-stage process. We first relate lattice quantities to the pole mass and then match the pole mass to the $\overline{\mathrm{MS}}$ mass evaluated at a scale equal to the $\mathrm{b}$ quark mass.

\subsection{Extracting the pole mass}

We determine the pole mass using two independent methods. The first method relates the pole mass, $M_{b}^{\text {pole }}$, to the experimental $\Upsilon$ mass, $M_{\Upsilon}^{\text {expt }}=9.46030(26) \mathrm{GeV}$ [11], using the heavy quark energy shift, $E_{0}$ :

$$
2 M_{b}^{\text {pole }}=M_{\Upsilon}^{\text {expt }}-\left(E^{\text {sim }}(0)-2 E_{0}\right) .
$$

Here $E^{\operatorname{sim}}(0)$ is the energy of the $\Upsilon$ meson at zero momentum, extracted from lattice NRQCD simulations. The quantity $\left(E^{\operatorname{sim}}(0)-2 E_{0}\right)$ corresponds to the 'binding energy' of the meson in NRQCD. We use a value of $E^{\mathrm{sim}}=0.515$ (3) GeV, obtained from a lattice NRQCD simulation run by the HPQCD collaboration on a "coarse" MILC ensemble, with lattice spacing $a=1.647$ (3) $\mathrm{GeV}^{-1}$ [12]. For further details of the configuration ensemble see [13, 14].

The second method directly matches the pole mass to the bare lattice mass in physical units, $M_{b}^{\text {latt }}(a)$, via the heavy quark mass renormalisation, $Z_{M}^{\text {latt }}$,

$$
M_{\mathrm{pole}}=Z_{M}^{\mathrm{latt}}\left(\mu a, M_{b}^{\mathrm{latt}}(a)\right) M_{b}^{\mathrm{latt}}(a)
$$

We employ a mixed strategy to calculate $E_{0}$ and $Z_{M}^{\text {latt }}$ perturbatively. The fermionic contributions to $E_{0}$, shown on the left-hand side of Figure 1, are calculated using two-loop automated lattice perturbation theory. All other contributions, shown on the right-hand side of Figure 1, are extracted from high-beta quenched simulations. 

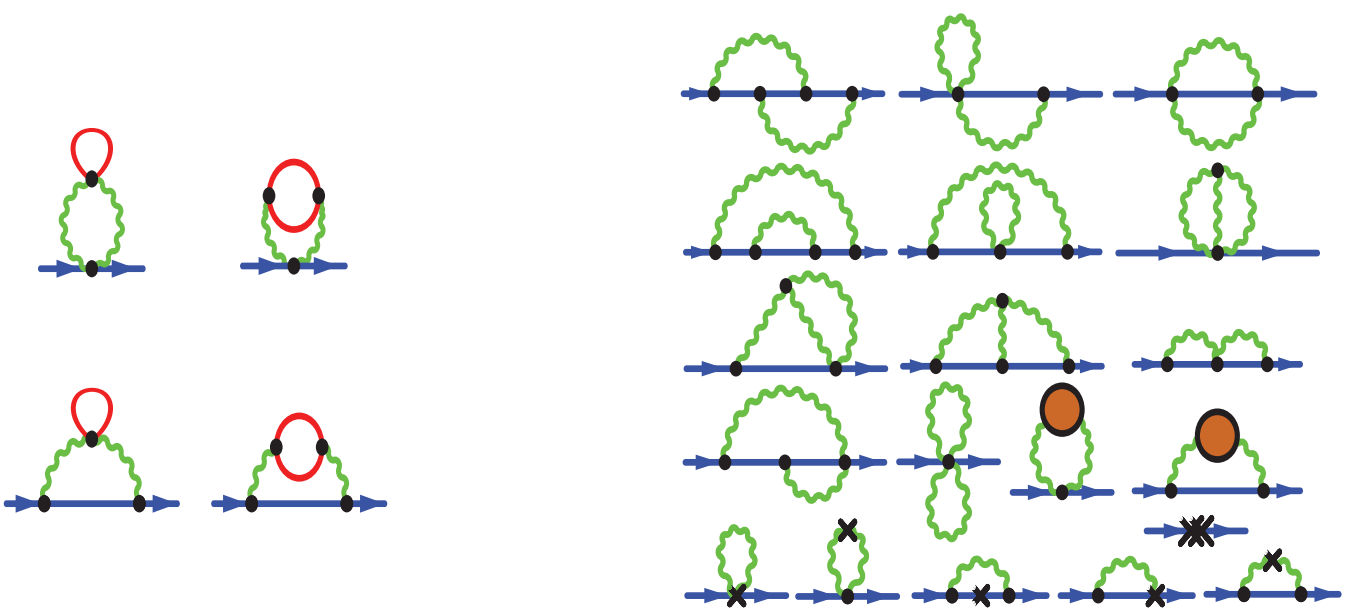

Figure 1: Contributions to $E_{0}$ and $Z_{M}^{\text {latt }}$. The four fermionic contributions calculated using automated lattice perturbation theory are shown on the left. The diagrams on the right are extracted from high- $\beta$ simulation. Blue lines are heavy quarks, green are gluons and red are sea quarks. Large brown blobs represent the 5 gluon self energy diagrams and crosses are counterterms. Feynman diagrams reproduced from [15].

Results were obtained using the NRQCD action of Equation 1.1 for the heavy valence quark, HISQ light quarks and the Lüscher-Weisz action for the gluons [16, 17]. We used a heavy quark mass in lattice units of $M a=2.8$, with a stability parameter of $n=2$.

\subsubsection{Automated lattice perturbation theory}

Feynman rules for the NRQCD and HISQ actions are too complicated to be viably derived by hand and the resulting Feynman integrals can only be evaluated numerically. We therefore use automated lattice perturbation theory, employing HiPPy to derive the Feynman rules and HP s rC to evaluate the four diagrams $[18,19]$. To control the highly-peaked IR behaviour of the Feynman integrands, we introduce a gluon mass. Although in general a non-zero gluon mass cannot be used in calculations beyond one-loop, this issue concerns only diagrams containing ghost-gluon vertices. In our calculation, these diagrams are handled by the high-beta simulation, allowing us to use a gluon mass for the fermionic contributions. The light quark diagrams in Figure 1 were calculated using five different light quark masses and extrapolated to zero light quark mass. We verified that the appropriate Ward identity for the 1-loop gluon self-energy was satisfied.

\subsubsection{High-beta simulations}

We perform quenched simulations on $L^{3} \times T$ lattices with temporal extent $T=3 L$, for $L=3$ to $L=10$ and twisted boundary conditions to reduce finite size effects and tunnelling between QCD vacua [17]. We generate ensembles of configurations for 17 values of $\beta$ from $\beta=9$ to $\beta=$ 120. Since the Green function is not gauge-invariant, we fix to Coulomb gauge using a conjugate gradient method. To extract the energy shift and mass renormalisation, we use an exponential fit to the heavy quark Green function parametrized as

$$
G(\mathbf{p}, t)=Z_{\psi} \exp \left(-\left[E_{0}+\frac{p^{2}}{2 Z_{M}^{\text {latt }} M_{0}}+\ldots\right] t\right),
$$


where the ellipsis stands for higher order terms that are included in the fits.

All operators in the NRQCD action are expressed in terms of gauge-covariant Wilson paths generated using PYTHON, which greatly enables flexibility and reduces programming errors. The heavy quark source is classified in the flavour-smell basis appropriate to the twisted boundary conditions. We implement the boundary conditions using a gauge-twist mask whenever a path in an operator crosses any spatial boundary. By applying an extra $U(1)$ phase in the mask, we can assign an arbitrarily small momentum to the source, enabling both $E_{0}$ and $Z_{M}^{\text {latt }}$ to be reliably extracted as a function $(\beta, L)$.

We convert $\beta$ to $\alpha_{V}$ and perform a joint fit to extract the 1- and 2-loop coefficients in the $L \rightarrow \infty$ limit. Simulations were run including tadpole improvement, which significantly reduces the magnitude of both 1- and 2-loop coefficients. Results for $Z_{M}^{\text {latt }}$ are good but this work is still in progress and we concentrate here on those for $E_{0}$. For even $L$, in Table 1 we compare the tadpoleimproved 1-loop coefficient from an unconstrained fit to the simulation data for $E_{0}$ with the exact result from automated perturbation theory. To extract the 2-loop coefficient we constrained the 1loop coefficient to be the exact value, but Table 1 shows that the simulation reliably reproduces the 1-loop results. The number of independent configurations for each $(\beta, L)$ was about 300, which we can easily increase by 10 -fold or more, allowing for much more accurate results at the next stage.

\begin{tabular}{|c||c|c|c|c|c|}
\hline$L$ & 4 & 6 & 8 & 10 & $\infty$ \\
\hline$E_{0}^{\text {sim. }}$ & $0.5295(16)$ & $0.5988(16)$ & $0.6369(12)$ & $0.6560(11)$ & $0.7380(63)$ \\
\hline$E_{0}^{\text {th. }}$ & 0.5312 & 0.6020 & 0.6362 & 0.6565 & $0.7348(3)$ \\
\hline
\end{tabular}

Table 1: Comparison of an unconstrained fit from simulation for the perturbative 1-loop coefficient with the automated perturbative calculation. There is no error on the theory calculation as it was done by mode summation. The error on the theory extrapolation to $L=\infty$ is estimated from a fit.

\subsection{Matching the pole mass to the $\overline{\mathrm{MS}}$ mass}

Although the pole mass is plagued by renormalon ambiguities, these ambiguities cancel when lattice quantities are related to the $\overline{\mathrm{MS}}$ mass. This renormalon cancellation is evident in the direct matching of the bare lattice mass to the $\overline{\mathrm{MS}}$ mass,

$$
M^{\overline{M S}}(\mu)=Z_{M}^{\text {latt }}\left(\mu a, M_{b}^{\text {latt }}(a)\right) Z_{\text {cont }}^{-1}\left(\mu, M_{\text {pole }}\right) M_{b}^{\text {latt }}(a),
$$

as both $M^{\overline{M S}}$ and $M_{b}^{\text {latt }}$ are renormalon-free. The continuum matching parameter, $Z_{M}^{\text {cont }}$, relates the pole mass to the $\overline{\mathrm{MS}}$ mass and has been determined to $\mathscr{O}\left(\alpha_{s}^{3}\right)$ [20].

To see that renormalon ambiguities also cancel in when determining the pole mass from the energy shift, we equate Equations 2.1 and 2.2 and rearrange them to obtain

$$
2\left(Z_{M}^{\text {latt }} M_{b}^{\text {latt }}(a)-E_{0}\right)=M_{\Upsilon}^{\text {expt }}-E^{\text {sim }}(0)
$$

The two quantities on the right hand side of the equation are renormalon ambiguity free: $M_{\Upsilon}^{\text {expt }}$ is a physical quantity and $E^{\operatorname{sim}}(0)$ is determined nonperturbatively from lattice simulations. Any renormalon ambiguities in the two power series, $Z_{M}^{\text {latt }}$ and $E_{0}$, on the left-hand side of the equation must therefore cancel. 


\section{Results}

For the fermionic and quenched contributions to the two-loop heavy quark energy shift we find

$$
E_{0}=0.7348(3) \alpha_{V}\left(q^{\star} / a\right)+\left(1.37(6)-0.0023(1) n_{f}\right) \alpha_{V}^{2}\left(q^{\star} / a\right)+\mathscr{O}\left(\alpha_{V}^{3}\right) .
$$

We express our result in the $V$-scheme at a scale $q^{\star} / a=3.33$, a value determined using the BLM procedure in [21]. The uncertainties quoted for the one-loop coefficient and the quenched contribution to the two-loop coefficient arise from the multi-polynomial fit. For the fermionic contribution to the two-loop coefficient, the quoted uncertainty is the statistical error in the numerical evaluation of the Feynman diagrams. We estimated the coefficient of the $\mathscr{O}\left(\alpha_{s}^{3}\right)$ term from the quenched simulation fits as $\sim 1.0(5)$.

Inserting this result for the heavy quark energy shift into Equation 2.1 leads to our first preliminary determination of the $\mathrm{b}$ quark mass:

$$
M^{\overline{M S}}\left(M^{\overline{M S}}\right)=4.25(12) \mathrm{GeV}
$$

The error is an estimate of $\mathscr{O}\left(\alpha_{s}^{3}\right)$ contributions, which dominate the uncertainty in our result. Uncertainties arising from systematic and statistical errors in the lattice results, $E^{\operatorname{sim}}(0)$ and $E_{0}$, are $\ll 1 \%$. We are unable to estimate the systematic error due to $O\left(a^{2}\right)$ artifacts as we have not yet finished the calculation for smaller values of $a$; this work is in progress and entails working with different values of $M a$ in NRQCD. It should be noted that we used a value of $E^{\operatorname{sim}}(0)$ that was generated from lattice NRQCD simulations using the action of Equation 1.1, but with $n=4$. From 1-loop calculations we estimate the errors associated with this mismatch to be much smaller than the dominant $\mathscr{O}\left(\alpha_{s}^{3}\right)$ error. However, this discrepancy will be corrected in future work.

\section{Conclusion}

We have calculated the two-loop heavy quark energy shift in highly-improved NRQCD using a mixed approach combining quenched high-beta simulations with lattice perturbation theory. This is the first determination of any heavy quark parameter beyond first-order perturbation theory in NRQCD, and demonstrates that we are able to extract a more precise prediction of the b quark mass from lattice NRQCD simulations than has been previously achieved. Work is currently underway to complete our calculation of the mass renormalisation, $Z_{M}^{\text {latt }}$, and to extend our results to incorporate different heavy quark masses to extrapolate to $a=0$. We also plan to increase the size of the ensembles used in the high-beta analysis by a significant factor. We expect these developments will improve further the precision of our result for the $b$ quark mass.

\section{Acknowledgements}

We would like to thank Christine Davies and Iain Kendall for providing HPQCD simulation data.

We thank the DEISA Consortium (www.deisa.eu), funded through the EUFP7 project RI222919, for support within the DEISA Extreme Computing Initiative. This work has made use of the resources provided by the Cambridge High Performance Computing service supported in part by the Science and Technology Facilities Council under grant ST/H008861/1. 


\section{References}

[1] E. Barberio, Inclusive semileptonic B decays, (2006) [arXiv: hep-ex/0605098]

[2] C. McNeile et al. (HPQCD), High-precision c and b masses, and QCD coupling from current-current correlators in lattice and continuum QCD, Phys. Rev. D 82 (2010) 034512 [arXiv: 1004 . 4285]

[3] K. G. Chetyrkin et al., Charm and bottom quark masses: an update, Phys. Rev. D 80 (2009) 074010 [arXiv:0907.2110 [hep-ph]]

[4] E. Follana et al. (HPQCD), Highly improved staggered quarks on the lattice with applications to charm physics, Phys. Rev. D 75 (2007) 054502 [arXiv: hep-lat / 0610092 ]

[5] A. Gray et al. (HPQCD \& UKQCD), The $\Upsilon$ spectrum and $m_{b}$ from full lattice $Q C D$, Phys. Rev. D 72 (2005) 094507 [arXiv: hep-lat/0507013]

[6] G. P. Lepage et al., Improved nonrelativistic QCD for heavy-quark physics, Phys. Rev. D 46 (1992) 4052

[7] R. R. Horgan et al., Moving nonrelativistic QCD for heavy-to-light form factors on the lattice, Phys. Rev. D 80 (2009) 074505 [arXiv: 0906.0945 ]

[8] G. T. Bodwin, Y-Q. Chen, Renormalon ambiguities in NRQCD operator matrix elements, Phys. Rev. D 60 (1999) 054008 [arXiv: hep-ph / 9807492 ]

[9] I. I. Bigi et al., Pole mass of the heavy quark: perturbation theory and beyond, Phys. Rev. D 50 (1994) 2234 [arXiv: hep-ph/9402360]

[10] M. Beneke, V.M. Braun, Heavy quark effective theory beyond perturbation theory: renormalons, the pole mass and the residual mass term, Nucl. Phys. B 426 (1994) 301 [arXiv: hep-ph/9402364]

[11] K. Nakamura et al. (Particle Data Group), Review of Particle Physics, J. Phys. G 37 (2010) 075021

[12] C. T. H. Davies, Private communication, 28/09/2010

[13] A. Bazavov et al., Full nonperturbative QCD simulations with 2+1 flavors of improved staggered quarks, Rev. Mod. Phys. 82 (2010) 1249 [arXiv : 0903.3598 ]

[14] C. T. H. Davies et al. (HPQCD), Precise determination of the lattice spacing in full lattice QCD, Phys. Rev. D 81 (2010) 034506 [arXiv: 0910 . 1229]

[15] Q. Mason, H. D. Trottier, R. R. Horgan, High precision fundamental constants using lattice perturbation theory, in proceedings of XXIIrd International Symposium on Lattice Field Theory, PoS (LAT2005) 011

[16] Zh. Hao et al., Unquenching effects on the coefficients of the Lüscher-Weisz action, Phys. Rev. D 76 (2007) 034507 [arXiv: 0 705.4660]

[17] M. Lüscher, P.Weisz, Efficient numerical techniques for perturbative lattice gauge theory calculations, Nucl. Phys. B 266 (1986) 309

[18] A. Hart et al., Automated generation of lattice QCD Feynman rules Comp. Phys. Comm. 180 (2009) 2698 [arXiv: 0904 .0375]

[19] T. C. Hammant et al., Improved automated lattice perturbation theory in background field gauge, in proceedings of XXVIII International Symposium on Lattice Field Theory

[20] K. Melnikov, T. van Ritbergen, The three-loop relation between the MS-bar and the pole quark masses, Phys. Lett. B 482 (2000) 99 [arXiv: hep-ph/9912391]

[21] K. Y. Wong, H. D. Trottier, R. M. Woloshyn, Perturbative Wilson loops from unquenched Monte Carlo simulations at weak couplings, Phys. Rev. D 73 (2006) 094512 [arXiv : hep-lat/0512012] 